\title{
Cytomegalovirus: Should We Screen Pregnant Women for Primary Infection?
}

\author{
Julie M. Johnson, MD ${ }^{1}$ Brenna L. Anderson, MD, MSc ${ }^{1}$ \\ ${ }^{1}$ Department of Obstetrics and Gynecology, Women and Infants \\ Hospital/Brown University, Providence, Rhode Island \\ Address for correspondence and reprint requests Julie M. Johnson, \\ MD, Clinical Assistant Professor, Department of Obstetrics and \\ Gynecology, Women and Infants Hospital/Brown University, 101 \\ Am J Perinatol 2013;30:121-124. \\ Dudley Street, Providence, RI 02905 (e-mail: jujohnson@wihri.org).
}

\begin{abstract}
Keywords

- congenital cytomegalovirus

- primary CMV infection

- CMV hyperimmune globulin

- screening program

Congenital cytomegalovirus (CMV) is a leading cause of neonatal morbidity, affecting $\sim 0.5$ to $1 \%$ of infants born each year. Primary maternal infection during early pregnancy is the greatest risk factor for severe neonatal morbidity/mortality. The current recommendation from national organizations advises against routine screening of pregnant women for primary infection. Recent advancements in diagnosis and treatment raise the issue of implementation of a national screening program. Prior to development of a major screening program for a highly prevalent and costly disease, the screening test must be safe, reliable, and valid with an effective and feasible intervention. This article reviews recent literature regarding available screening tests and potential interventions and whether criteria for a screening program are met in the current state of science. Although screening women using CMV immunoglobulin (Ig) G, $\operatorname{lgM}$, and $\lg G$ avidity testing is reliable, effective intervention with hygiene modification or treatment with CMV-specific hyperimmune globulin is not as well established. More evidence from randomized controlled trials is needed prior to moving forward with a screening program for congenital CMV.
\end{abstract}

Congenital cytomegalovirus (CMV) is the most common cause of congenital infection in the United States, with a prevalence of $0.64 \% .^{1,2}$ About 1 in every 150 babies born in the United States is infected, with a total of 30,000 cases each year. ${ }^{3}$ Almost 8,000 children are permanently disabled with symptoms such as developmental delay, deafness, and vision loss each year, and almost 400 die every year as a result of congenital CMV. ${ }^{4,5}$ This translates into $\sim 2$ billion dollars spent on CMV-related health care costs per year in addition to immeasurable emotional burden to those affected. $^{5}$

Given this tremendous impact on society, research in the area of prevention and treatment of primary maternal CMV infection is a priority. Recent developments for potential prevention strategies, including CMV-specific hyperimmune globulin (HIG) and hygiene modification, in addition to new screening tools such as immunoglobulin (Ig) G avidity assays, make screening for primary maternal CMV infection tempt- ing. However, before recommending screening for CMV as a routine part of prenatal care, one must consider whether the test meets established criteria for the introduction of a screening test.

\section{Criteria for Implementation of CMV Screening}

Due to recent developments in diagnosing and preventing maternal and congenital infection, there has been some national discussion regarding the issue of screening for primary maternal infection. ${ }^{6,7}$ Prior to instituting a formal guideline for screening all pregnant women, it is important to assess whether key criteria for disease screening are met. The disease must be clinically important, prevalent, and well characterized. The screening test must be safe, reliable, and valid. Finally, the intervention must be effective, cost-effective, and feasible. received

August 14, 2012

accepted after revision

November 1, 2012

published online

December 29, 2012
Copyright $\odot 2013$ by Thieme Medical Publishers, Inc., 333 Seventh Avenue, New York, NY 10001, USA. Tel: +1(212) 584-4662.
DOI http://dx.doi.org/ 10.1055/s-0032-1333133. ISSN 0735-1631. 


\section{Epidemiology}

Maternal infection with CMV can be primary or nonprimary. Nonprimary infections may represent reactivation of a latent infection or reinfection with a new strain of virus. In reproductive age women, seroprevalence rates range from 40 to $83 \%^{8,9}$ A primary maternal infection, as evidenced by seroconversion during pregnancy, occurs in about 1 to $4 \%$ of pregnant women, which results in approximately 27,000 cases per year in the United States. ${ }^{5,10-12}$ In women with a nonprimary infection, the rate of fetal transmission is $1 \% .{ }^{13}$ In contrast, the rate of fetal transmission in women who seroconvert during pregnancy is 30 to $40 \%{ }^{14}$ As gestational age increases, the rate of vertical transmission increases. However, fetal infection early in pregnancy is associated with worse sequelae. $^{15}$

Transmission of CMV occurs through direct contact with the bodily fluids, such as saliva, urine, or semen, of someone who is actively shedding the virus. For women of reproductive age, the greatest risk for exposure is through contact with the urine or saliva of young children. ${ }^{16}$ This places women with young children or working in daycare centers at a higher risk of infection. ${ }^{17}$

\section{Biology of Placental CMV Infection}

Most immunocompetent women infected with CMV are asymptomatic. If women develop symptoms, they are usually vague and consist of fever, malaise, myalgias, and chills. ${ }^{18}$ Once infected, IgM and IgG antibodies to CMV are produced. The IgM antibody titer is usually high for the first 1 to 3 months (acute phase) and then declines thereafter (convalescent phase). ${ }^{19}$ IgM antibodies may persist for up to 6 to 9 months after primary infection and may be present with nonprimary infection. ${ }^{20}$ Once the virus is present in the maternal bloodstream, neutralizing antibodies bind with viral antigen. Neutralizing $\operatorname{IgG}$ antibodies are those that bind with high avidity. Those $\operatorname{Ig} G$ antibodies with low avidity are indicative of a recent infection and have poor neutralizing capability. One theory of the mechanism of placental infection is through transcytosis of a low avidity IgG-virion complex into the syncitiotrophoblast. ${ }^{21}$ Low-avidity IgG antibodies are present only with primary infection and may be detectable for 3 to 5 months after initial exposure. ${ }^{22}$ The exact mode of fetal transmission is unknown.

\section{Current Recommendations for Screening}

The most recent recommendation from the American College of Obstetricians and Gynecologists (ACOG) is to not routinely screen patients for CMV. Due to the fact that many women acquire the infection through contact with young children, ACOG does recommend that women who are in close contact with young children (mothers or daycare workers) be educated regarding transmission and hygiene practices to reduce transmission. ${ }^{18}$ The Centers for Disease Control (CDC) also does not recommend routine maternal screening. ${ }^{23}$ The CDC recommends education regarding hygiene practices aimed at preventing transmission of the virus, such as washing hands after changing a diaper and not sharing utensils or food with young children. ${ }^{24}$ At this time, there are no high-quality studies demonstrating efficacy of hygiene measures during pregnancy.

\section{Screening Tests for Primary CMV Infection}

The gold standard for diagnosis of a primary infection is demonstration of seroconversion with CMV IgG antibodies. ${ }^{22}$ Since routine screening in low-risk women is not routinely recommended, seroconversion is rarely documented. Screening with serial titers is not feasible because it would require monthly blood tests on every seronegative pregnant woman.

Primary infection may also be suspected by the presence of CMV IgM antibodies. If IgM antibodies are present, the infection may be acute. However, they may also be present in the convalescent phase or nonprimary infections. In addition, false-positive results can occur as a result of crossreactivity with other disease entities, such as parvovirus B19 or systemic lupus erythematosus. There are several commercially available diagnostic kits for CMV IgM and none are standardized for antigen composition. Therefore, discrepant results can occur among the different testing kits. $^{25-27}$

With such uncertainty surrounding a positive IgM, it can be difficult to determine whether an infection is primary. Recently, the IgG avidity test has become available and is able to detect an acute infection with 92 to $100 \%$ sensitivity and 82 to $100 \%$ specificity. ${ }^{20}$ When combined with a true positive IgM, a low/moderate IgG avidity test has the same diagnostic value as seroconversion. ${ }^{20}$

The avidity test determines whether the $\operatorname{IgG}$ antibody has high avidity, indicating previous infection, or low avidity, indicating a primary infection. It takes about 18 to 20 weeks for the IgG antibody to demonstrate high avidity after an acute infection. Therefore, a low avidity test prior to the 18th to 20th weeks of pregnancy can identify those women at high risk of severe congenital infection. ${ }^{20}$ Using the approach of a one-time test, either with an initial serology screen or not, would be more feasible and cost-effective than serial IgG testing.

\section{Intervention}

The ideal intervention for prevention of congenital CMV would be a vaccine. However, an effective and safe vaccine is still years away from reality, so efforts at prevention have also focused on maternal education regarding hygiene and potential treatment with CMV HIG.

Given that congenital CMV infection affects more babies than Down syndrome, neural tube defects, or fetal alcohol syndrome, one may think the general public would be aware of its presence. A recent survey by Cannon et al suggests otherwise. Only $13 \%$ of women and $7 \%$ of men surveyed had heard of congenital CMV. Given that transmission of the virus is through contact with bodily fluids, educating pregnant women regarding the mode of transmission and hygiene 
practices that may prevent viral acquisition seems plausible as a means to reduce maternal infection.

There have been preliminary studies examining hygiene modification. ${ }^{28-30}$ In 1996, Adler et al evaluated 36 nonpregnant and pregnant seronegative women who received education, education with documented adherence, or no education. ${ }^{28}$ Although the sample size was insufficient to show a benefit of education, none of the pregnant women in either education group seroconverted. This study suggested that pregnant women may be more motivated to modify behavior in an attempt to avoid infection. In 2004, Adler again evaluated pregnant and nonpregnant seronegative women randomized to behavior modification versus control. ${ }^{29} \mathrm{Al}-$ though there was no difference in seroconversion rates between the two groups, educated pregnant women again were less likely to become infected than their nonpregnant counterparts. Picone et al performed a prospective cohort study of high-risk, seronegative women and the impact of education and hygiene information. ${ }^{30}$ Although there were low rates of seroconversion, there was no control group. Currently, there are inadequate data to show that education actually changes behavior and that this behavior change translates into decreasing maternal infection and subsequent congenital infection.

A second topic of research regarding prevention of congenital disease is aimed at prevention of maternal-fetal transmission. There have been preliminary results to suggest that treatment with HIG after documentation of a primary maternal infection may reduce the risk of congenital infection. A nonrandomized study by Nigro et al of 181 women with primary infection demonstrated the potential for HIG to be used as a means of prevention of fetal infection. ${ }^{31}$ The prevention group consisted of 37 women who did not undergo amniocentesis because of gestational age $<20$ weeks or proximity of diagnosis (within 6 weeks of primary infection), or they simply declined. This group was offered $100 \mathrm{U} / \mathrm{kg}$ of HIG every month until delivery. The comparison group was 47 women who did not undergo amniocentesis but also declined prevention therapy. In the prevention group, $16 \%$ of infants whose mothers received HIG had congenital infection versus $40 \%$ of infants in the group declining HIG. However, controversy exists regarding the heterogeneity of the prevention group, lack of randomization, and small sample size of this study.

Recently, preliminary data from a randomized trial of CMV HIG for prevention was negative, with a $44 \%$ rate of congenital infection in the placebo group versus $30 \%$ rate in the treatment group $(p=0.13){ }^{32}$ The full results have not yet been published. There is also one other large, multicenter randomized trial underway in the United States by the Maternal-Fetal Medicine Units Network, which is expected to complete enrollment in 2016 ("A Randomized Trial to Prevent Congenital Cytomegalovirus Infection," ClinicalTrials.gov \# NCT01376778). Until completion of this trial, it is not known whether CMV HIG is effective or safe.

\section{Conclusion}

Congenital CMV is a major public health problem. Efforts at reducing the rate of infection are a top priority of obstetric research. Although significant advancements have been made, there are still several knowledge gaps regarding intervention. In this age of scrutiny regarding public health policies and the cost-effectiveness of screening and intervention, care must be taken to fully evaluate a screening program with regard to the disease, the test, and the intervention prior to full implementation.

In the case of CMV, several conditions have been met, although others remain missing. The disease itself is clinically important, well defined, and prevalent. Due to years of diagnosis without an available treatment, the natural history is known, with substantial morbidity/mortality if left untreated. In addition, the development of the avidity assay has made it possible to detect asymptomatic disease.

With evidence that the disease fulfills criteria for screening, the next step is to evaluate the screening test itself. The screening technique of using either seroconversion or $\operatorname{IgG}$, IgM antibodies in addition to IgG avidity is well described. The test is itself is safe and reliable. It is not known at this time whether it would be accepted by pregnant women. Given that such a small percentage of the population is even aware of this condition, maternal perception of the risks and benefits of screening and treatment cannot be adequately assessed at this time.

The final consideration regarding a screening program is the cost-effectiveness and feasibility of the proposed intervention. A recent cost-effectiveness model by Cahill et al examined possible screening strategies for primary maternal infection. ${ }^{6}$ Universal screening, screening in high-risk women, screening with fetal findings on ultrasound, and a baseline reference of no screening or treatment were compared. Using decision analysis models to assess neonatal outcomes with CMV HIG treatment, universal screening appeared to be the most cost-effective strategy. However, these findings are not based on data from a randomized controlled trial. More studies regarding cost-effectiveness are needed once the current trials are completed.

Screening tests for maternal primary infection of CMV have improved over the last decade, and if employed in the correct manner, could be used in a very cost-efficient way. However, screening programs would have to be developed once treatment is shown to be effective. For example, if a large-scale trial of seronegative pregnant women demonstrated the effectiveness of behavior modification at preventing maternal acquisition, serological screening early in pregnancy would be rational. In addition, if the CMV HIG proves itself to be safe and effective at preventing vertical transmission, testing for primary maternal infection with antibodies and IgG avidity in the second trimester has proven reliable in identifying those at risk. However, neither of these interventions has proven to be effective in large randomized trials, and neither should be accepted as standard of care until shown to be effective and safe. In the meantime, the recommendations of ACOG and the CDC should be followed. We should continue to educate those at risk about the virus and test those with symptoms of active disease. It is not yet time for universal serum screening for maternal CMV infection. 


\section{References}

1 Kenneson A, Cannon MJ. Review and meta-analysis of the epidemiology of congenital cytomegalovirus (CMV) infection. Rev Med Virol 2007; 17:253-276

2 Grosse SD, Ross DS, Dollard SC. Congenital cytomegalovirus (CMV) infection as a cause of permanent bilateral hearing loss: a quantitative assessment. J Clin Virol 2008;41:57-62

3 Centers for Disease Control and Prevention. Congenital CMV trends and statistics. Available at: http://www.cdc.gov/cmv/ trends-stats.html. Accessed December 21, 2012

4 Arvin AM, Fast P, Myers M, Plotkin S, Rabinovich R; National Vaccine Advisory Committee. Vaccine development to prevent cytomegalovirus disease: report from the National Vaccine Advisory Committee. Clin Infect Dis 2004;39:233-239

5 Cannon MJ, Davis KF. Washing our hands of the congenital cytomegalovirus disease epidemic. BMC Public Health 2005;5:70

6 Cahill AG, Odibo AO, Stamilio DM, Macones GA. Screening and treating for primary cytomegalovirus infection in pregnancy: where do we stand? A decision-analytic and economic analysis. Am J Obstet Gynecol 2009;201:e1-e7

7 Adler SP. Screening for cytomegalovirus during pregnancy. Infect Dis Obstet Gynecol 2011;2011:1-9

8 Gaytant MA, Steegers EAP, Semmekrot BA, Merkus HM, Galama JM. Congenital cytomegalovirus infection: review of the epidemiology and outcome. Obstet Gynecol Surv 2002;57:245-256

9 Staras SAS, Dollard SC, Radford KW, Flanders WD, Pass RF, Cannon MJ. Seroprevalence of cytomegalovirus infection in the United States, 1988-1994. Clin Infect Dis 2006;43:1143-1151

10 Stagno S, Pass RF, Dworsky ME, Alford CA Jr. Maternal cytomegalovirus infection and perinatal transmission. Clin Obstet Gynecol 1982;25:563-576

11 Ludwig A, Hengel H. Epidemiological impact and disease burden of congenital cytomegalovirus infection in Europe. Euro Surveill 2009;14:26-32

12 Colugnati FAF, Staras SAS, Dollard SC, Cannon MJ. Incidence of cytomegalovirus infection among the general population and pregnant women in the United States. BMC Infect Dis 2007;7:71

13 Fowler KB, Stagno S, Pass RF. Maternal immunity and prevention of congenital cytomegalovirus infection. JAMA 2003;289:1008-1011

14 Stagno S, Pass RF, Cloud G, et al. Primary cytomegalovirus infection in pregnancy. Incidence, transmission to fetus, and clinical outcome. JAMA 1986;256:1904-1908

15 Enders G, Daiminger A, Bäder U, Exler S, Enders M. Intrauterine transmission and clinical outcome of 248 pregnancies with primary cytomegalovirus infection in relation to gestational age. J Clin Virol 2011;52:244-246

16 Cannon MJ, Westbrook K, Levis D, Schleiss MR, Thackeray R, Pass RF. Awareness of and behaviors related to child-to-mother transmission of cytomegalovirus. Prev Med 2012;54:351-357

17 Centers for Disease Control and Prevention. Cytomegalovirus (CMV) and congenital CMV infection. At-risk patients. Available at: http://www.cdc.gov/cmv/risk/infants-children.html. Accessed December 21, 2012
18 ACOG. ACOG Practice Bulletin \#20. Perinatal viral and parasitic infections. ACOG 2011

19 Revello MG, Gerna G. Diagnosis and management of human cytomegalovirus infection in the mother, fetus, and newborn infant. Clin Microbiol Rev 2002;15:680-715

20 Lazzarotto T, Guerra B, Lanari M, Gabrielli L, Landini MP. New advances in the diagnosis of congenital cytomegalovirus infection. J Clin Virol 2008;41:192-197

21 Maidji E, McDonagh S, Genbacev O, Tabata T, Pereira L. Maternal antibodies enhance or prevent cytomegalovirus infection in the placenta by neonatal Fc receptor-mediated transcytosis. Am J Pathol 2006;168:1210-1226

22 Dollard SC, Staras SAS, Amin MM, Schmid DS, Cannon MJ. National prevalence estimates for cytomegalovirus IgM and IgG avidity and association between high IgM antibody titer and low IgG avidity. Clin Vaccine Immunol 2011;18:1895-1899

23 Centers for Disease Control and Prevention. Cytomegalovirus (CMV) and congenital CMV infection. Testing and diagnosis of CMV infection. Available at: http://www.cdc.gov/cmv/testingdiagnosis.html. Accessed December 21, 2012

24 Centers for Disease Control and Prevention. Cytomegalovirus (CMV) and congenital CMV infection. Preventing congenital CMV infection. Available at: http://www.cdc.gov/cmv/prevention.html. Accessed December 21, 2012

25 Maine GT, Stricker R, Schuler M, et al. Development and clinical evaluation of a recombinant-antigen-based cytomegalovirus immunoglobulin $M$ automated immunoassay using the Abbott AxSYM analyzer. J Clin Microbiol 2000;38: 1476-1481

26 Lazzarotto T, Galli C, Pulvirenti R, et al. Evaluation of the Abbott AxSYM cytomegalovirus (CMV) immunoglobulin M (IgM) assay in conjunction with other CMV IgM tests and a CMV IgG avidity assay. Clin Diagn Lab Immunol 2001;8:196-198

27 Revello MG, Genini E, Gorini G, Klersy C, Piralla A, Gerna G. Comparative evaluation of eight commercial human cytomegalovirus IgG avidity assays. J Clin Virol 2010;48:255-259

28 Adler SP, Finney JW, Manganello AM, Best AM. Prevention of childto-mother transmission of cytomegalovirus by changing behaviors: a randomized controlled trial. Pediatr Infect Dis J 1996; $15: 240-246$

29 Adler SP, Finney JW, Manganello AM, Best AM. Prevention of childto-mother transmission of cytomegalovirus among pregnant women. J Pediatr 2004;145:485-491

30 Picone O, Vauloup-Fellous C, Cordier AG, et al. A 2-year study on cytomegalovirus infection during pregnancy in a French hospital. BJOG 2009;116:818-823

31 Nigro G, Adler SP, La Torre R, Best AM; Congenital Cytomegalovirus Collaborating Group. Passive immunization during pregnancy for congenital cytomegalovirus infection. N Engl J Med 2005;353: $1350-1362$

32 Adler SP. Editorial commentary: primary maternal cytomegalovirus infection during pregnancy: do we have a treatment option? Clin Infect Dis 2012;55:504-506 